\title{
A study on the planning concept of walking for health referring to "Tokyo Hoken Doro Promenade Proposal"
}

\author{
J. Sanada \\ Department of Civil Engineering, the University of Tokushima, Japan
}

\begin{abstract}
Walking is regarded as important for a healthy society. But in Japan, the situation is not good. People living in cities walk under skyscrapers in automobile exhaust, and even in suburban areas it is hard to find safe roads which are relatively free from traffic. Thus, this paper looks at "Hoken Doro Promenade" as a new type of walkway which was proposed for tranquillity and peace of mind. "Hoken Doro" means a promenade for health which was planned before WWII. This paper aims to clarify the concept of design of Hoken Doro Promenade and its spatial meaning. For this purpose, the procedure of this study consists of the following three steps. First, the formation process of Hoken Doro Promenade is given. Next, the founder of this (Tokutaro Kitamura) is introduced. Third, by means of rearranging his descriptions, the concept of design of Hoken Doro Promenade is defined. The knowledge of Hoken Doro Promenade that was obtained from this study is as follows: 1) Kitamura proposed promenades running along a river, but they differed from the other promenades of that time in 3 ways; (a) located in suburbs, (b) designed for pedestrians, (c) tried to construct with a minimum of artificial work. 2) They aimed to make the riverside open and maintain the natural environment by setting the promenade along the river. 3) They were planned in scenic zones, so the design was considered taking into account the scenery of fields, 4) It was enjoyed that promenades would play a role not only as the introducer of famous scenes but also to promote non famous ones. In conclusion, it can be said that, Hoken Doro Promenade was an institution that is considered to the surrounding area's features and the pedestrian. From the results of this study, the author proposes the value of walking trail networks in modern cities.
\end{abstract}

Keywords: park system, walking trail, health. 


\section{Introduction}

Walking is regarded as important for a healthy society. But in Japan, the situation is not good. People living in cities walk under skyscrapers in automobile exhaust, and even in suburban areas it is hard to find safe roads which are relatively free from traffic. Thus, this paper looks at "Hoken Doro Promenade" as a walking trail which was proposed for tranquillity and peace of mind.

Hoken Doro Promenade is a pedestrian way which was planned for the suburban areas of Tokyo City. First it was officially released with the name as "Tokyo Hoken Doro" in 1938, and the next year it was incorporated in the Tokyo Park System with some alterations. The Tokyo Park System is a regional park system extending over Tokyo, Kanagawa, Saitama and Chiba Prefectures. It is an infrastructural plan containing a network of park ways, regional parks, block parks and greenbelt (this is composed of circular part and wedge-shaped parts, and is planned for recreational use.).

With the above in mind, it is apparent that Hoken Doro is not a simple promenade; it is planned as a part of regional planning. In addition, Hoken Doro is explained as being a new promenade because it is completely for pedestrians, and does not follow the Street Structure Law, has varying widths and is not permitted to be paved even with ballast, in its plan. But up to the present, almost all of the plan of Hoken Doro has not been realized.

This paper aims to clarify the concept of design of Hoken Doro Promenade and its spatial meaning, and then discuss planning of walking trail networks.

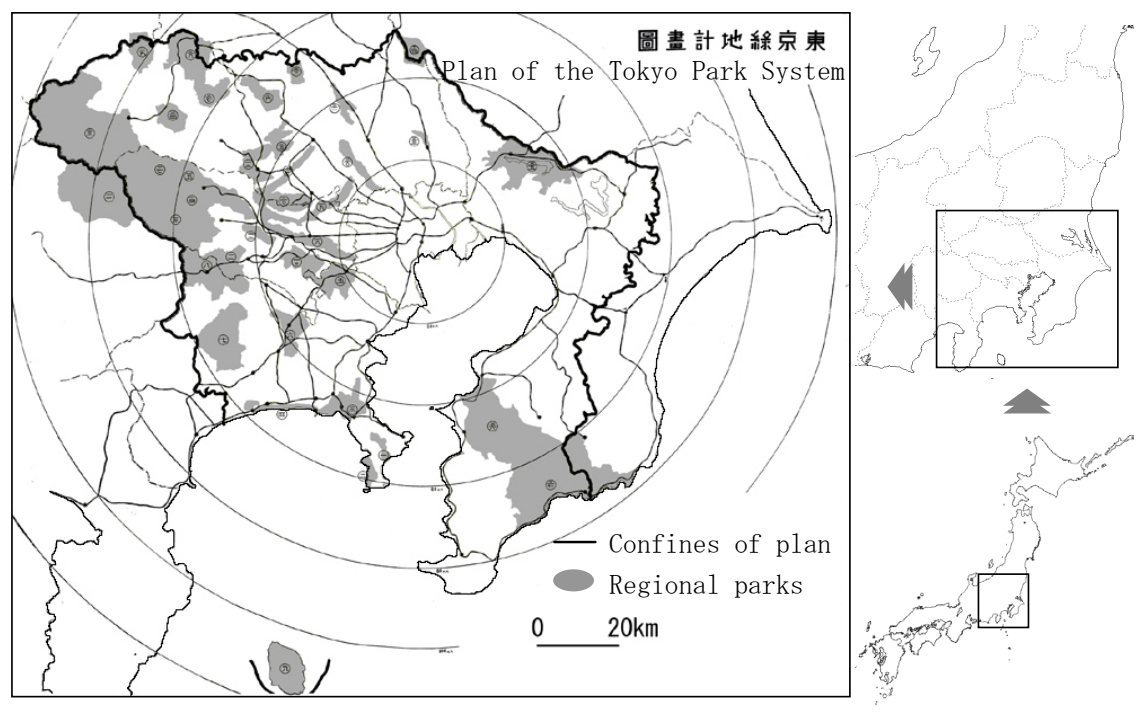

Figure 1: $\quad$ Plan of the Tokyo Park System and its location in Japan [1]. 


\section{Outline of Hoken Doro}

We can recognize from the plan and the names of each route of Hoken Doro that it is planned along rivers. The aim is quoted as follows:

"The mission of Hoken Doro is to promote peoples' health by walking." [2]

To show the necessity of Hoken Doro, the death rate in Japan is compared with that of European and American countries. It is observed that people must be healthy in order to have an efficient military and labour force. In addition, it is clear that walking is both easy and good for our health. Ultimately walking and Hoken Doro may be treated related to the strength of a nation.

It is possible to take these explanations as the function of Hoken Doro. However, it should be understood that when Hoken Doro was first planned, Japan was a country at war where travel for personal pleasure was not permitted, but travel related to fostering imperialistic sentiment was allowed. Therefore, it may be hasty to judge that Hoken Doro was simply for health but rather had ulterior motives.

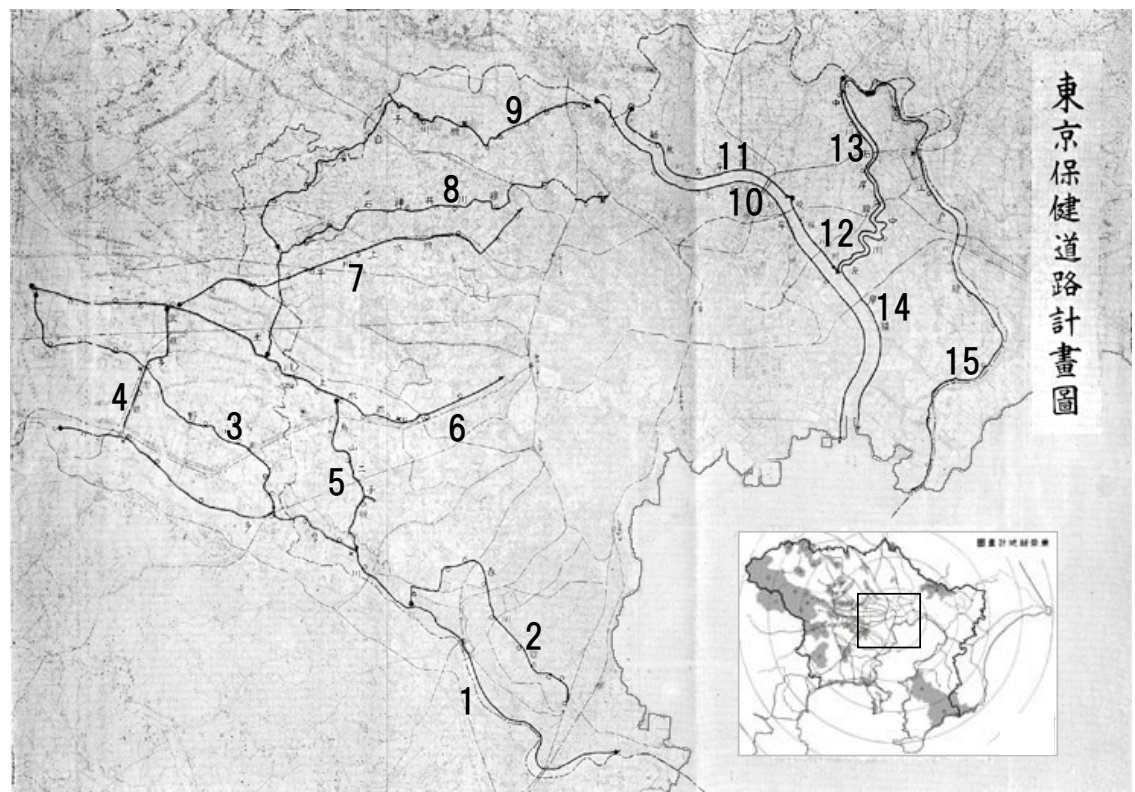

1. Tamagawa River Way

2. Nomigawa River Way

3. Nogawa River Way

4. Musashi-Tama Way

5. Karasuyama-Futako Way

6. Tamagawa-Josui Canal Way

7. Senkawa-Josui Canal Way

8. Syakujiigawa River Way
9. Shirakogawa River Way

10. The Right Bank of New Arakawa River Way

11. The Left Bank of New Arakawa River Way

12. Ayasegawa River Way

13. The Right Bank of Nakagawa River Way

14. The Left Bank of Nakagawa River Way

15. Edogawa River Way

Figure 2: $\quad$ Plan of Tokyo Hoken Doro, in 1938 [3]. 


\section{Hoken Doro and Tokutaro Kitamura}

Hoken Doro differs from other promenades of that time. It is the idea of the person who proposed it, namely, Tokutaro Kitamura. Kitamura was engineer of the Home Office, and was the primary planner of the Tokyo Park System. Kitamura came to contribute articles to a magazine specialised in city planning from 1923 [5].

\subsection{The deal of conservation of Riparian ways in Germany}

Here, it is necessary to introduce one article that Kitamura transrated and introduced German open space planning [4].

In this article, Kitamura introduces German law of 1922: "Das Gesetz zur Erhaltung des Baumbestandes und Erhaltung von Uferwegen im Interesse der Folksgesundheit (The law about conservation of trees and conservation of riparian ways in the interest of the nation's health)" and the Declaration of 1911 which was announced by the governor of County of Potsdam.

According to the article, the aim of the law was to select riparian ways to be conserved which can be helpful related to the nation's health and recreation. In the notation of the law, it is said that the law is not aimed at construction of roads, but rather is aimed at preventing riverside from becoming inaccessible due to being occupied by houses. In addition, it is said that the reason why the law was made was because trips to rivers is beneficial to one's health.

Also, in the declaration of 1911 it is mentioned that the scenic riverside can easily become occupied by rich people's villas, but these areas should be preserved for the public. To make protect such riversides, local government should make plan of riparian ways before such land is sold. And regarding these ways along the side of the river, should have a pedestrian way of small width and no roadway.

That is, in Germany riparian ways the aim is to save the riversides which are beneficial for both health and recreation of walkers. So establishing ways is a means for this purpose; accordingly small-scaled pedestrian way is preferential to large roadway construction. From looking at the previous discussion, we can see the foundation of Hoken Doro.

\subsection{Kitamura's view about Riparian ways}

In this section, I discuss Kitamura's view about riparian ways from his articles. Kitamura wrote about waterside areas as follows:

"In scenic areas the riverside will be occupied by houses in most areas. I propose to conserve linear areas which have around 1 meter width along the river. What I propose is not a roadway. I believe that it does not place occupants at a disadvantage" [6].

"Riverside in suburban areas is suitable for pedestrians. Japanese cities have scarce parks and scarce open spaces, with only riverside areas having abundant trees and grass" [7]. 
"Even a small width area of riparian ways can facilitate to satisfy requirements for walking for pleasure. And setting these ways is easer than construction of parks. So I propose planning riparian ways with surrounding scenic areas before such areas become occupied by houses. And I suggest that the ways are connected to each other if possible" [8].

The following 6 points are recognized from Kitamura's words quoted above:

(1) Riverside should be preserved for people with pedestrian ways constructed.

(2) Establishing riparian ways is effective for conservation or tree and grassland.

(3) Riparian ways satisfy requirements for walking just as parks do.

(4) It is preferable that riparian ways are designed as only pedestrian ways with a small width.

(5) Riparian ways, if possible, should be planned so as to connect them to other recreational areas such as parks or open spaces.

(6) It is preferable that riparian ways are connected to each other.

We should notice that these articles ware not written about Hoken Doro, but ware written about riverside or riparian ways in around 1930 before Hoken Doro was planned.

\section{The concept of Hoken Doro from design, layout and descriptions}

This chapter clarifies the concept of Hoken Doro from conditions of design, layout and each description of routes.

\subsection{The concept of Hoken Doro from design}

It is said that the aim of Hoken Doro is walking for physical training, but then it is said that ways are set choosing areas which include places with clean air, nice scenery, comfortable waterside, fine forest and peaceful field. From these, it is indicated that Hoken Doro was not simply for walk but was for pleasure.

In conditions of design, it is said "A major part of routes pass in scenic zone, so it is important not to spoil the environment and to harmonize it with the surroundings". Related to the above, the following conditions are presented [9].

(1) Hoken Doro must be a pedestrian way with a width of 2 meters or more: depending on landform.

(2) Structure of each way doesn't follow the Street Structure Law.

(3) Efforts should be made to reduce cut and fill by fixing longitudinal slope on present land slope.

(4) Consider drainage in detail.

(5) Ways with the exception of some areas such as a mud should not be paved even with ballast.

(6) Avoid damage to natural surroundings, especially avoid cutting down trees. 
(7) When new areas of trees or bushes are planed or added to existing foliage, they should harmonize with the present surrounding area.

(8) Put mini parks every 2 kilo meters as rest areas for walkers.

(9) Utility poles or similar structures should be prohibited on ways.

(10) Existing utility poles or other structures must be relocated to areas outside of the way.

Established species of tree or fixed numerical targets are not shown in the above 10 conditions, but rather, the idea that design by study on surroundings; present trees, land slope etc is outlined. What is stressed in the above is that, it is important to execute the plan with minimal damage on the surrounding environment.

\subsection{The idea of Hoken Doro from its plan}

It is said that Hoken Doro is planned for the suburban areas of Tokyo City. We will now examine its layout more closely, focusing on the relationship between Hoken Doro and rivers, greenbelt and parks. It should be noted that greenbelt does not aim at restricting urban expansion but aims at promoting recreation, in Japan. As for its shape, it is composed of circular part and wedge-shaped parts.

To understand the relationships of them, here I use the plan of 1939 which was published as a part of the Tokyo Park System. This plan has 24 routes.

The following 5 points are identified from the layout:

(1) A total of 23 routes are located along the river.

(2) All of routes access another route. 10 routes connect at both ends, and 12 routes connect at one end.

(3) 17 routes pass parks with 9 routes of them starting from parks.

(4) 13 routes pass circular part or wedge-shaped parts of green belt. As for 9 routes of them, the entire route is located in green belt.

(5) 9 routes arrive at greenbelt. Overall, 22 routes including 13 routes above are related to greenbelt.

We can recognize from these facts that Hoken Doro, parks and greenbelt are interconnected with each other.

\subsection{The concept of Hoken Doro from descriptions of routes}

In the Plan of Hoken Doro, an explanation is provided for each route [11]. Here I will examine them. Explanation starts with fundamental information such as location, length, width and mini parks contained. Some cross section is added, too. And in a major part of the explanation, there is description detailed of surroundings and view. It is described in detail, and is written with a focus on the fine points of scenery. It is like a guidebook of walking. It is an interaction of both walking and seeing as the scenery continually changes; it is not limited to simple explanation of routes. From a fact that a major part of explanation is description detailed like this, it follows that the aim of Hoken Doro is to take pleasure in walking whilst enjoying scenery. 


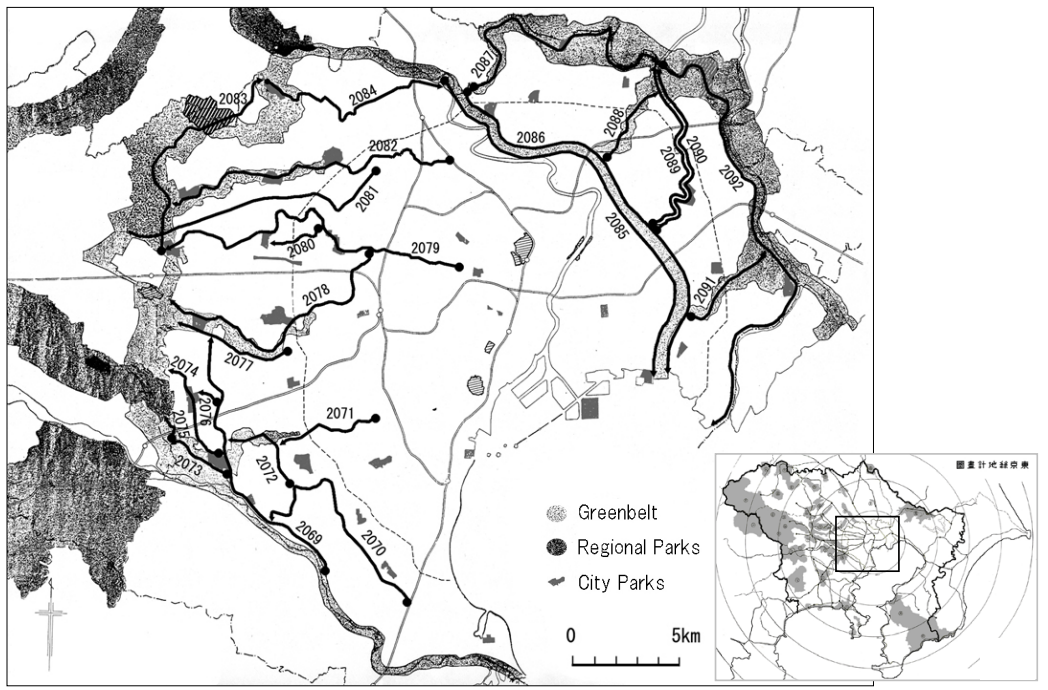

Figure 3: $\quad$ Hoken Doro and Parks and Green Belt, in 1939 [10].

Then, what scenery do planners hope walkers see? Descriptions of scenery can be divided roughly into two groups; one is a group of famous scenic sites, and the other is a group of nameless sites. Famous sites may include well known shrines, temples, old trees, famous mountains such as Mt. Fuji and so on. Nameless sites include lush green vegetation, red leaves, wild grass, wildflowers etc. In addition to these plants seasonal sounds produced by chirps of rail,

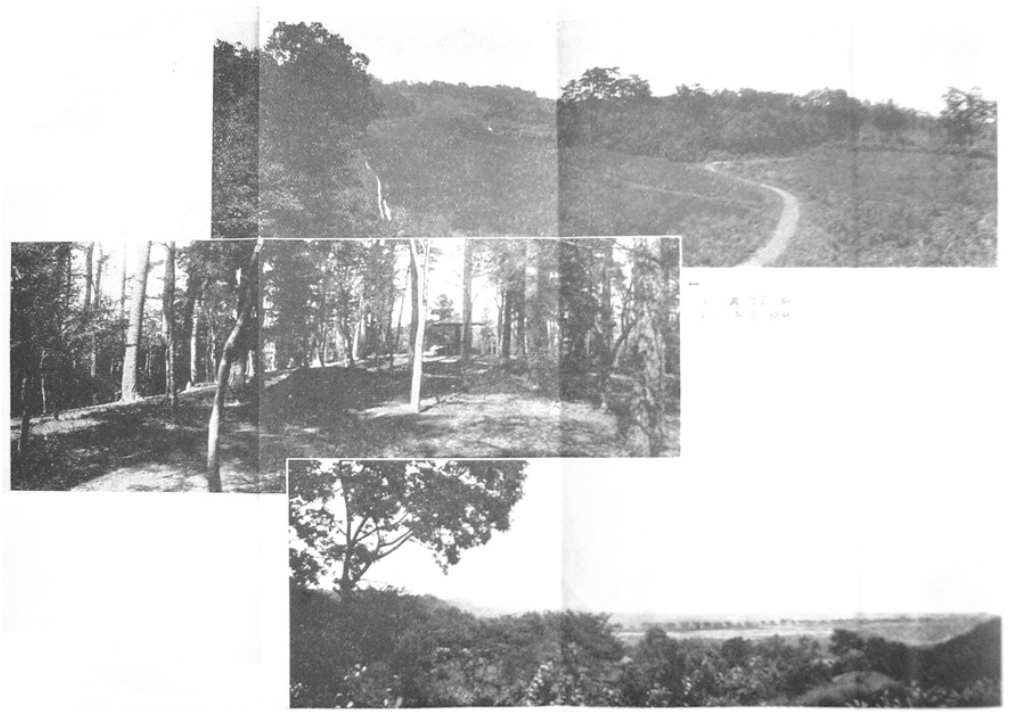

Figure 4: $\quad$ Photos attached to the plan of Hoken Doro [12]. 
Japanese bush warbler, "bell-ringing" cricket and pine cricket. And eyes stop on expanses of the rice paddies, meandering river and terrace range. Moreover, taste is found from living scene such as a farmer making pickles in his yard. It is recognized that different type of scenery is mentioned.

And then I will discuss a visual distance to objects looked at. Not only nearby scenery such as flowers along the way is here but also for example, rice paddies not so far away stretching to distant scenes such as Mt. Fuji.

From these observations we may conclude that planners intended people to walk and enjoy the scenery. So Hoken Doro may be completed with surroundings sceneries of even distant site and with people who walks there and looks these sceneries.

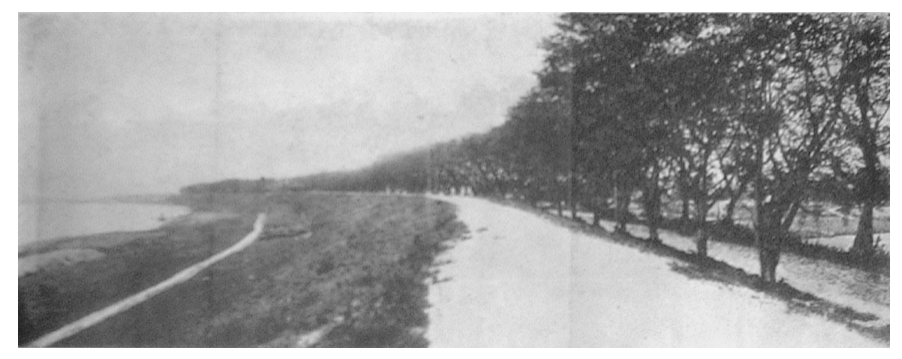

Figure 5: $\quad$ Photo attached to the plan of Hoken Doro [12].

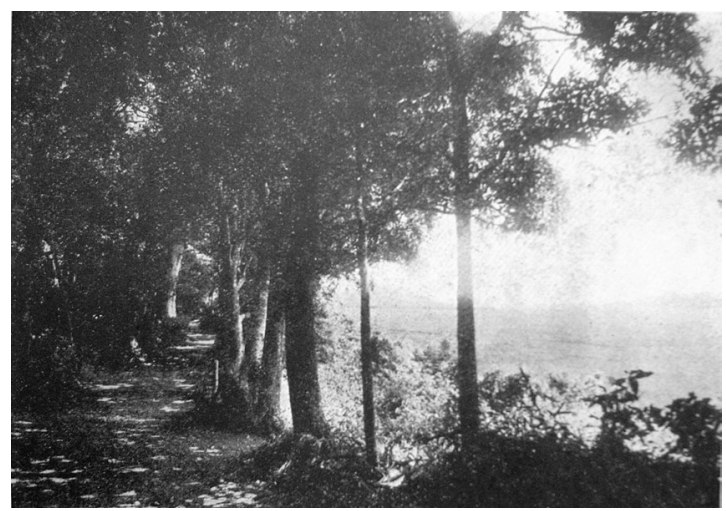

Figure 6: $\quad$ Photo attached to the plan of Hoken Doro [12].

\section{Conclusion}

Hoken Doro was proposed by Tokutaro Kitamura. He referred to German riparian ways. The ideas of German ones are "the aim is walking for health", "walk along the riverside is a good form of recreation", "establishing ways along rivers allows the waterside to be open for public and also serves to conserve such 
areas", "there is no aim to construct magnificent roads". In addition, Kitamura had such ideas as "riparian ways can be planned so as to connect them to other recreational areas such as parks or open spaces" and "it is preferable that they are networked with each other".

From conditions of design and layout of the Plan of Hoken Doro, it can be seen that the ideas above are realized. And from descriptions of routes, we can see that planners intended people to walk and enjoy the scenery.

I considered the above and propose such ideas for present-day walking trails. It is important that walking trails are safe from traffic and bad pavement such as pot holes, bumps etc. In addition to these elements of structure, it is important consider layout on the scale of the whole city. That is to say, it is important that walking trails are networked with each other and connected to other recreational areas in a city. But to plan for only their network is not sufficient, I suggest considering surroundings and views together, so it is important where we select. After considering such things, it will become practicable to make walking trails which people want to walk on.

\section{References}

[1] Parks and reservations, 3(2.3), frontispiece, 1939.

[2] Tokyo Regional Planning Commission, The Plan of Tokyo Hoken Doro, Fundamental Issues of Urban Planning, 2, p.148, 1938.

[3] Fundamental Issues of Urban Planning, 2, p.160, 1938.

[4] Tokutaro, K., Legislation about parks and open space in Germany, Discussions on City Planning, 8(7), pp.31-43, 8(7), pp.26-38, 1925.

[5] The magazine is Discussions on City Planning, articles are for example, 6(7) of 1923, 7(7) of 1924, 8(7)(8) of 1925, 10(4)(7)(8) of 1927 etc.

[6] Tokutaro, K., Planning and Scenery of Resort, Discussions on City Planning, 13(7), p.69, 1930.

[7] Tokutaro, K., Photos of Current Parks and Reservations in the World, Discussions on City Planning, 14(8), p.309, 1931.

[8] Tokutaro, K., About Scenic Zone, Discussions on City Planning, 10(4), p.12, 1931.

[9] Tokyo Regional Planning Commission, The Plan of Tokyo Hoken Doro, Fundamental Issues of Urban Planning, 2, pp.150-151, 1938.

[10] Parks and reservations, 3(2.3), frontispiece, 1939.

[11] Tokyo Regional Planning Commission, The Plan of Tokyo Hoken Doro, Fundamental Issues of Urban Planning, 2, pp.151-165, 1938.

[12] Tokyo Regional Planning Commission, The Plan of Tokyo Hoken Doro, Fundamental Issues of Urban Planning, 2, pp.160-161, 1938. 